\title{
Influencia de variables físicas en la productividad de Pinus arizonica y Pinus engelmannii en el sur de Chihuahua, México
}

\author{
Influence of physical variables in the yield of Pinus arizonica \\ and Pinus engelmannii in the south of Chihuahua, México
}

\author{
Martín Martínez-Salvador, ${ }^{1 *}$ Ricardo David Valdez-Cepeda ${ }^{2}$ \\ y Marín Pompa García ${ }^{3}$
}

\begin{abstract}
RESUMEN
Se utilizó el índice de sitio (IS) para identificar la influencia de variables de suelo, clima y relieve sobre el crecimiento de $P$. arizonica y $P$. engelmannii en el sur de Chihuahua. Los modelos de IS fueron desarrollados a partir de información de análisis troncales usando la función de Schumacher y el método de curva guía. Adicionalmente, se desarrolló un muestreo sistemático usando parcelas de $400 \mathrm{~m}^{2}$, donde se midieron altura y edad de árboles dominantes, así como variables de suelo, clima y relieve. Con los modelos de IS se identificó la calidad de estación de cada parcela muestreada. Se realizó un análisis de discriminante canónico (ADC), para identificar diferencias multivariadas entre calidades de estación, y un análisis de varianza, para identificar la influencia de las variables físicas en el crecimiento de las especies. El método de curva guía predijo con precisión el crecimiento de ambas especies (Pseudo $\mathrm{R}^{2}>0,90$ ). El ADC mostró que existen diferencias multivariadas entre las calidades de estación, con alta influencia de la pendiente, disección vertical, profundidad de suelo y temperaturas. De acuerdo con el análisis de varianza $(\alpha \leq 0,05)$, los valores para las variables de mayor influencia sobre la mejor calidad de estación fueron la profundidad de suelo $>43 \mathrm{~cm}$, pendiente menor a $12^{\circ} \pm 8^{\circ}$, temperatura mínima promedio menor a $8{ }^{\circ} \mathrm{C} \pm 3{ }^{\circ} \mathrm{C}$ y temperatura máxima promedio menor a $18^{\circ} \mathrm{C} \pm$ $3^{\circ} \mathrm{C}$. Estos valores pueden ser de gran utilidad para construir modelos espaciales de productividad en los estados de Chihuahua y Durango, México.
\end{abstract}

PALABRAS CLAVE

Calidad de estación, crecimiento, índice de sitio, variables físicas.

\begin{abstract}
In the south of Chihuahua Mexico, site index (IS) models were used in order to identify the influence of soil, climate and topography variables on $P$. arizonica and $P$ engelmannii growth. The IS models were fitted by using the Schumacher equation and the guide curve with information from stems analysis. Additionally a systematic sampling method was carried out on $400 \mathrm{~m}^{2}$ field plots. Dominant tree height and age, and physical variables of the sites were measured. Site index models were adjusted with tree height and age data for both species. With site index models the productivity of each sampled plot was identified. An analysis of canonical discriminate was carried on in order to identify multivariate differences in the productivity levels, and an analysis of variance was conducted to detect differences per each variable studied. Site index models accuracy were statistically significant with a pseudo $r^{2}>0,9$. The ADC showed differences in the levels of productivity, where slope, vertical dissection, soil deep and temperatures were the most important variables. According to the analysis of variance $(\alpha \leq 0,05)$, the
\end{abstract}


values for places whit highest productivity, and those variables which have more influence over the growth of both species are: soil depth $>43 \mathrm{~cm}$, slope lower than $12^{\circ} \pm 8^{\circ}$, average of minimum temperature of $8{ }^{\circ} \mathrm{C} \pm 3{ }^{\circ} \mathrm{C}$, maximum temperatures $<18{ }^{\circ} \mathrm{C} \pm 3{ }^{\circ} \mathrm{C}$. These values could be highly useful to build spatial models of productivity in the northwest of Mexican forest.

KEYWORDS

Station quality, growth, site index, physical variables.

\section{INTRODUCCIÓN}

En los bosques de la Sierra Tarahumara del sur de Chihuahua, México, crecen poblaciones nativas de Pinus arizonica $y$ Pinus engelmannii, las cuales son aprovechadas para la extracción de madera. El manejo sustentable de los bosques requiere del conocimiento de los factores ambientales que determinan el crecimiento de las especies forestales (Palahí et al., 2004). La influencia de estos factores sobre la producción y productividad del bosque generalmente se evalúa a través de la construcción de modelos de índice de sitio, como una medida indirecta de la calidad de estación (Madrigal, 2002); sin embargo, en pocas ocasiones se evalúa la influencia directa de las variables físicas sobre el crecimiento del arbolado (Heiberg y White, 1956; Martínez et al., 2006).

La calidad de estación se refiere a la capacidad inherente de un territorio para hacer crecer los árboles que sostiene y usualmente se utilizan modelos que relacionan edad-altura de árboles dominantes para calificarla. Estos modelos son conocidos como índice de sitio (Clutter et al., 1983), los cuales están determinados por la suma de los factores como suelo, topografía, clima y factores bióticos, que interrelacionados afectan la productividad de un bosque (Spurr, 1952; Spurr y Barnes, 1982; Clutter et al., 1983; Daniel et al.,
1982; Musalem et al., 1986; Pritchett, 1986; Deo, 2013).

En trabajos realizados en México se describen las relaciones entre la altitud y la distribución de especies de pino (Eguiluz, 1977; Eguiluz, 1982; Madrigal, 2002) y recientemente se han desarrollado modelos bioclimáticos para reconocer patrones de distribución potencial de especies de pinaceas y para la cuantificación de características climáticas donde las especies prosperan o podrían desarrollarse con mayor productividad (Téllez et al., 2004; Peters et al., 2013).

Existe un creciente interés por conocer la influencia de variables físicas sobre el crecimiento de especies y productividad de los bosques (Martínez, 2008; Medlyn et al., 2011), ya que el conocimiento de esta información es importante para generar cartografía de potencial productivo de diferentes áreas mediante el uso de herramientas de Sistemas de Información Geográfica (SIG) (Martínez et al., 2006; Latta et al., 2009).

Estudios ecológicos muestran que se han encontrado relaciones entre el crecimiento de coníferas y la profundidad de suelo, altitud, pendiente y exposición (Castaños, 1962; Griffiths et al., 2009). Otros trabajos mencionan que las propiedades físicas que más influyen en el crecimiento de algunas coníferas son $\mathrm{pH}$, material muerto y contenido de limo en la capa de $50 \mathrm{~cm}$ de suelo (Rodríguez, 1982; Stoler y Relyea, 2011). Estos antecedentes pueden ser usados para construir modelos espaciales a partir de valores de óptimas condiciones para el desarrollo de las especies (Sánchez y Chacón, 1986; Martínez, 2008).

Con la finalidad de caracterizar la influencia de variables físicas de los factores de clima, suelo y relieve en los bosques del sur de Chihuahua, México, se 
clasificaron sitios con diferente productividad (alta, media y baja), usando modelos de índice de sitio. Los resultados son de gran utilidad para generar y validar cartografía de los bosques en el norte de México y para implantar mejores prácticas de manejo en los predios en los que se realiza cosecha de madera.

\section{MATERIALES Y MÉTODO}

El estudio se realizó en los bosques del municipio de Guadalupe y Calvo, en la Sierra Tarahumara del sur de Chihuahua, México en los años 2006 y 2007 (Fig. 1).

El municipio de Guadalupe y Calvo se encuentra entre los $26^{\circ} 41^{\prime}$ y $25^{\circ} 34^{\prime}$ de latitud norte y $106^{\circ} 25^{\prime}$ y $107^{\circ} 42^{\prime}$ de longitud oeste, tiene una superficie aproximada de 904000 hectáreas. El clima que predo- mina en el municipio es templado subhúmedo con lluvias en verano (García, 1981). Los principales suelos del municipio son litosol, regosol, xerosol, feozem, cambisol y planosol (CETENAL, 1972); los cuales, se caracterizan por ser muy delgados y con alto porcentaje de pedregosidad. Los tipos de vegetación de la región son bosques templados, selva baja caducifolia, pastizales y matorrales (Rzedowsky, 1978). La principal actividad productiva en el municipio es la silvicultura con una cosecha anual de aproximadamente $300000 \mathrm{~m}^{3}$ de madera en rollo, lo cual representa $25 \%$ de la madera que se produce en el estado y $3,7 \%$ de la producción nacional.

Para el ajuste de modelos de índice de sitios se realizaron análisis troncales a un grupo de 40 árboles dominantes de $P$. arizonica y 42 de $P$. engelmannii siguiendo

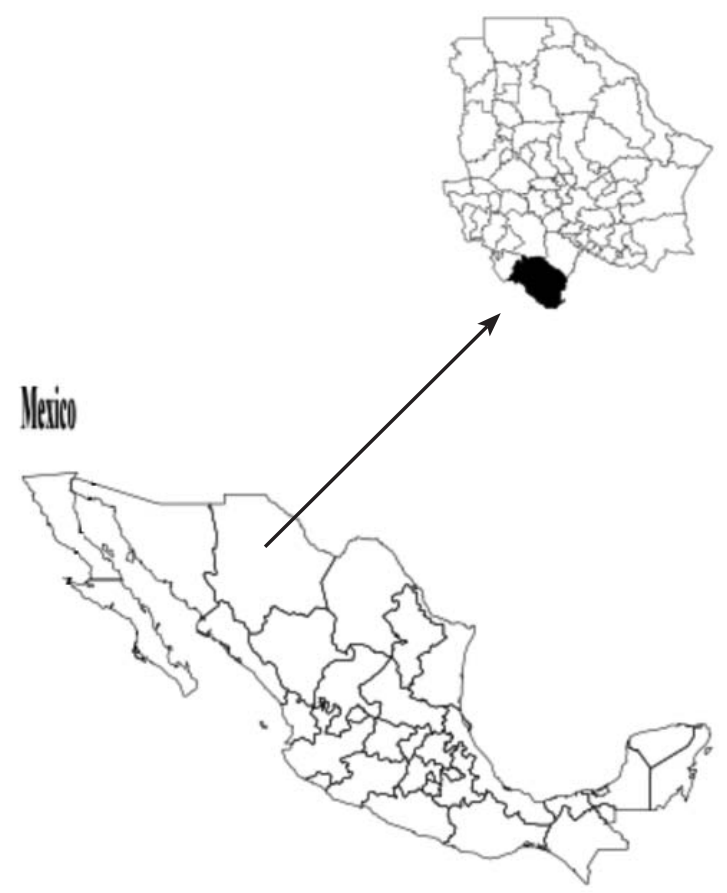

Figura 1. Área de estudio. 
las metodologías propuestas por Mas (1970) y el FPC (1998); las cuales consisten en examinar secciones transversales del fuste, seleccionadas a $30 \mathrm{~cm}$, $1,30 \mathrm{~m}$ y cada $2 \mathrm{~m}$ hasta alcanzar $10 \mathrm{~cm}$ de diámetro, con medición de la punta. Las secciones transversales fueron analizadas mediante el conteo y medición de anillos para integrar la base de datos con información de edad, diámetro y altura para el posterior análisis mediante procedimientos de regresión no lineal.

Para calificar la calidad de estación en los bosques del municipio se muestrearon 220 sitios circulares de $400 \mathrm{~m}^{2}$ distribuidos en un diseño sistemático de $5 \mathrm{~km}$ entre sitios. En cada sitio se anotaron los datos de ubicación geográfica y la altura sobre el nivel medio del mar, y se tomó información de altura y edad de tres árboles dominantes de las especies $P$. arizonica y $P$. engelmannii. Además se midieron variables físicas de los factores suelo, relieve y clima.

Para el caso del suelo se midieron la profundidad de suelo $(\mathrm{cm})$, pedregosidad (\%), suelo desnudo (\%), grosor de la capa de mantillo (grosor de mantillo) (cm), grosor de la capa de hojarasca (grosor de hojarasca) en centímetros. Para el relieve se midieron la pendiente $\left({ }^{\circ}\right)$, exposición (Azimut), disección horizontal (densidad de arroyos por kilómetro cuadrado) y disección vertical (diferencia en altura sobre el nivel del mar en $\mathrm{m} / \mathrm{km}^{2}$ ), las dos últimas variables se derivaron del análisis del Modelo Digital de Elevación esc 1:50 000 .

Finalmente, se obtuvieron datos de variables de clima como la temperatura mínima, temperatura máxima y precipitación. Las variables de clima se generaron mediante modelos de interpolación en SIG con información de estaciones meteorológicas ubicadas en la región de influencia del área de estudio que comprende los estados de Durango, Chihuahua, Sonora y Sinaloa (IMTA, 2006).

\section{Análisis de la información}

Con la información de altura y edad proveniente de los análisis troncales se ajustaron funciones de índice de sitio para las especies Pinus arizonica y Pinus engelmannii utilizando el modelo de Schumacher con el método de curva guía.

Modelo Schumacher

$$
H=\beta_{0} e^{-\beta_{1} E}
$$

donde:

$H=$ Altura dominante $(\mathrm{m})$

$E=\operatorname{Edad}$ (años)

$\beta_{0}=$ Parámetros de los modelos

Cada sitio de muestreo fue calificado de acuerdo con su calidad de estación utilizando la información de edad y altura de los árboles dominantes de las parcelas de muestreo y los modelos de índice de sitio ajustados para ambas especies.

Se establecieron tres niveles de productividad (calidad de estación) I. Alta, II. Media y III. Baja, y posteriormente se realizó un análisis de discriminante canónico (ADC) para detectar diferencias multivariadas entre las tres calidades de estación a partir de la base de datos de variables físicas.

Se consideró el método de la distancia cuadrada Mahalanobis y la prueba de Lamda Wilks como estadísticos de prueba para la toma de decisiones sobre las funciones de discriminante canónico (FDC) (Hair et al., 1998).

Una vez identificadas diferencias multivariadas entre calidades de estación, 
y posterior a una estandarización de la base de datos, se realizó un análisis de varianza para identificar diferencias a nivel de variables individuales, con especial atención en las variables que definieron las funciones de discriminante canónico (FDC). Finalmente, se realizó una comparación de medias usando el procedimiento de Tukey con un valor de significancia de 0,05.

Para el análisis de varianza se usó un modelo completamente aleatorio.

$$
Y_{I}=M+T_{i}+\mathrm{x}_{j}
$$

donde:

$Y_{i}=$ variable resultado del modelo

$M_{i}=$ media generalizada

$T_{i}=$ efecto de tratamiento (calidad de estación)

$X_{i k j}=$ error experimental.

\section{RESULTADOS Y DISCUSIÓN}

Los modelos de índice de sitio para $P$. arizonica, y $P$. engelmannii fueron estadísticamente aceptables (Tabla 1) y altamente predictivos con una pseudo $\mathrm{R}^{2}$ de 0,98 para $P$. arizonica y 0,92 para $P$. engelmannii. Estos modelos permitieron clasificar la base de datos de sitios de muestreo en función de tres calidades de estación (niveles de productividad) (Figs. 2 y 3 ). Los valores observados que se muestran en las figuras 2 y 3 corresponden a las alturas de los árboles dominantes medidos en campo en los sitios de muestreo, mientras que las curvas corresponden a los modelos de IS ajustados con el modelo de Shumacher, usando el método de curva guía a partir de pares de datos edad-altura de los análisis troncales.

Estos modelos son útiles para predecir el crecimiento de los árboles en el futuro y de esta manera realizar adecuadamente la planificación del manejo, proyecciones del inventario y determinación del potencial de cosecha (Clutter et al., 1983; Palahí et al., 2004; Martínez et al., 2007); sin embargo, con estos modelos es importante construir cartografía, la cual permitirá integrar con mayor facilidad el índice de sitio a la planeación y manejo de bosques, por lo que es necesario conocer la influencia de las variables ecológicas como suelo, pendiente y relieve sobre el crecimiento de las especies (Spurr y Barnes,1982; Martínez, 2008).

Una de las pruebas que resultan de gran utilidad para identificar diferencias en la influencia de variables o grupos de variables sobre las diferentes condiciones

Tabla 1. Modelos de Schumacher de índice de sitio ajustados por el método de curva guía.

\begin{tabular}{|c|c|c|c|c|c|c|}
\hline \multirow{2}{*}{ Model } & \multicolumn{2}{|c|}{ Estimadores } & \multicolumn{2}{c|}{ Int. Confid $\beta_{1}$} & $\begin{array}{c}\text { Pseudo } \\
R^{2}\end{array}$ & Pr $>F$ \\
\cline { 2 - 5 } & $\beta_{0}$ & $\beta_{1}$ & Inf. & Sup. & & \\
\hline Pinus arizonica & 26,98 & 30,96 & 20,40 & 41,53 & 0,98 & 0,0001 \\
\hline Pinus engelmannii & 26,52 & 34,44 & 22,03 & 46,84 & 0,92 & 0,0001 \\
\hline
\end{tabular}




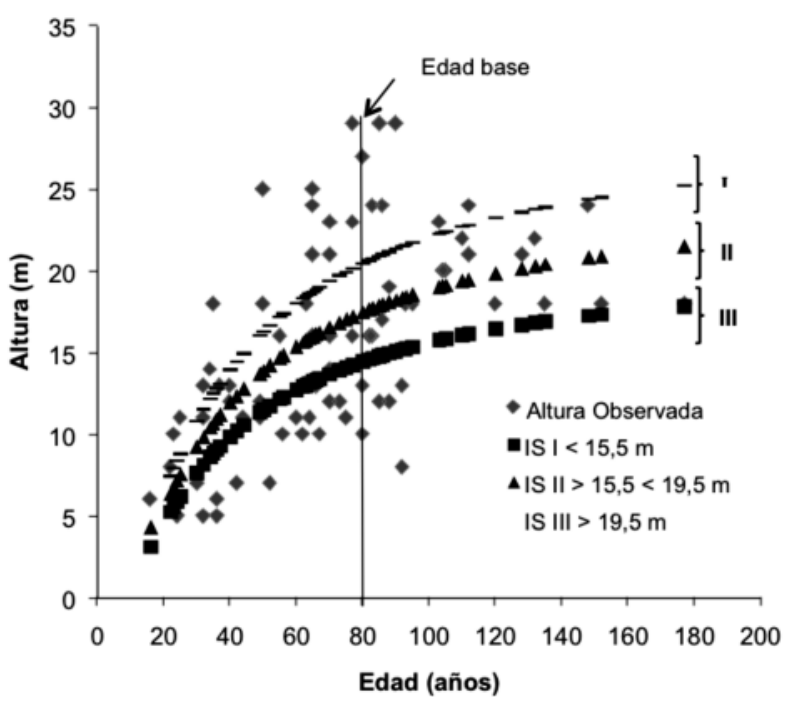

Figura 2. Modelo de Schumacher con el método de curva guía en Índice de Sitio de Pinus arizonica Engelm., en Guadalupe y Calvo, Chihuahua, México.

Se analizó el crecimiento de los árboles y se estableció la clasificación de las curvas de índice de sitio (IS) a una edad base de 80 años. En función de la edad base el IS $<15,5 \mathrm{~m}$ representa la curva de los sitios de productividad baja que agrupa a los árboles que alcanzan una altura de $15,5 \mathrm{~m}$, el IS $>15,5<19,5$, representa la curva de los sitios de productividad media que agrupa a los árboles que alcanzan alturas entre $15,5 \mathrm{~m}$ y $19,5 \mathrm{~m}$ y el IS > 19,5 m, representa la curva de los sitios de productividad alta que agrupa a los árboles que alcanzan alturas superiores a los 19,5 m.

de un sistema es el análisis de determinante canónico (ADC). En este estudio al realizar el ADC, la distancia cuadrada de Mahalanobis indicó que las calidades de estación son diferentes entre sí $(P<0,05)$. Por su parte, la prueba de Lambda Wilks también mostró diferencias multivariadas entre las tres calidades de estación $(P<0,05)$.

La correlación canónica y el vector raíz indicaron que la primer correlación canónica fue mayor que la segunda $(P<0,05)$ con un coeficiente de correlación $\mathrm{R}=0,50$, y un valor del vector raíz de 0,60 , mientras que el porcentaje de la variación explicada con la primer función discriminante fue de 0,83 .
La estructura canónica total (Tabla 2) muestra que las variables pedregosidad, pendiente, disección vertical y exposición están negativamente relacionadas con la primera variable canónica, mientras que las variables altura sobre el nivel del mar (asnm), profundidad de suelo, temperatura máxima y mínima, mantillo y precipitación, están relacionadas positivamente. La primer función de discriminante canónico muestra que la separación entre niveles de productividad está dada por la disección vertical $(R=0,60)$, precipitación $(\mathrm{R}=0,48)$, profundidad de suelo $(R=0,43)$ y la pendiente $(R=0,28)$.

El análisis de discriminante canónico indica que las calidades de estación son 


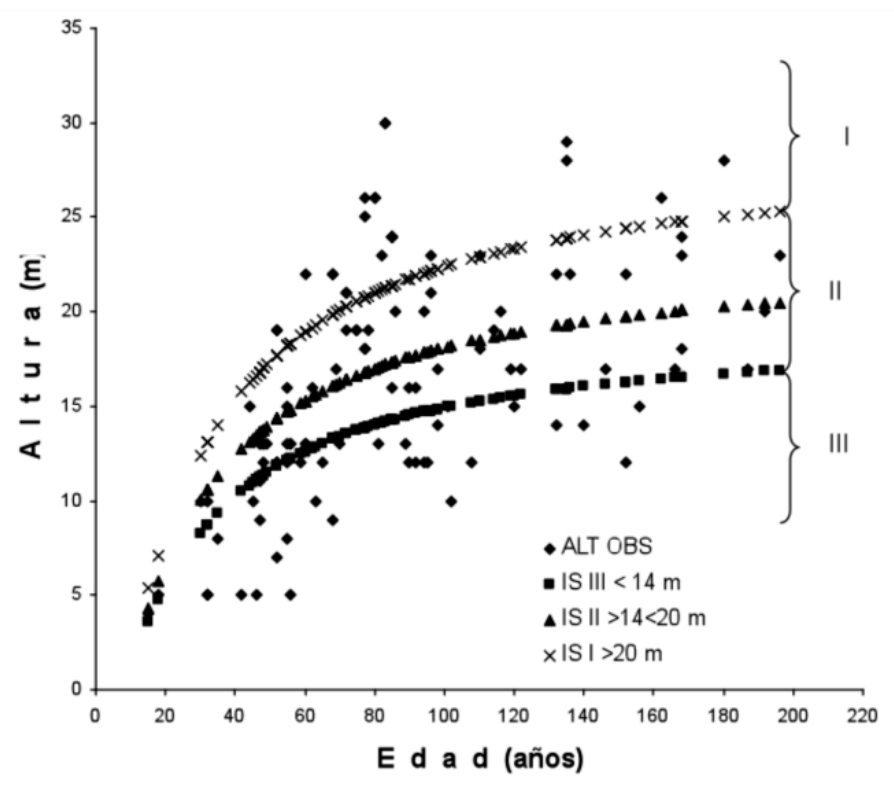

Figura 3. Modelo de Schumacher con el método de curva guía en Índice de Sitio de Pinus engelmannii en Guadalupe y Calvo, Chihuahua, México.

Se analizó el crecimiento de los árboles, y se estableció la clasificación de las curvas de índice de sitio (IS) a una edad base de 80 años. En función de la edad base el IS $<14 \mathrm{~m}$, representa la curva de los sitios de productividad baja que agrupa a los árboles que alcanzan una altura de $14 \mathrm{~m}$, el IS $>14<20$ representa la curva de los sitios de productividad media que agrupa a los árboles que alcanzan alturas entre $14 \mathrm{~m}$ y $20 \mathrm{~m}$ y el IS>20 m, representa la curva de los sitios de productividad alta que agrupa a los árboles que alcanzan alturas superiores a los $20 \mathrm{~m}$.

multivariadamente distintas $(P<0,005)$, considerando la primer función canónica (Fig. 4). Por su parte, la figura 5 muestra gráficamente estas diferencias entre las tres calidades de estación.

Con base en el DCA se observa mayor influencia de las variables topográficas y climáticas sobre la productividad. Al respecto, estudios recientes muestran que las características climáticas influyen considerablemente sobre la productividad del bosque, donde las temperaturas juegan un papel importante en la generación de microambientes que aceleran la descomposición del material orgánico y la consecuente liberación de nutrientes; mientras que las relaciones entre la precipitación y las densidades de arbolado incrementan la disponibilidad de humedad en bosques secos (Deo, 2013; Raz-Yaseef et al., 2010; Karhu et al., 2010; Latta et al., 2010), como los de la región de estudio.

Una vez identificadas diferencias entre las calidades de estación, es importante conocer la diferencia de cada variable entre las calidades de estación con la finalidad de identificar el grupo de varia- 
bles que podrían apoyar al entendimiento espacial de la productividad.

Los resultados del análisis de varianza realizado al grupo de variables físicas estima que la variable asnm no tuvo diferencias significativas $(\alpha \leq 0,05)$ entre los sitios con diferente productividad (Tabla 3). Al respecto, Spurr y Barnes (1982) y Eguiluz (1982) mencionan que los factores ambientales que más influyen sobre la productividad del bosque son los climáticos, edáficos y biológicos, mientras que la altura, aunque influye en la delimitación del área óptima de desarrollo de las especies, tiene una mayor influencia sobre su distribución.

La pendiente, disección vertical, exposición, temperatura mínima, temperatura máxima y profundidad de suelo presentaron diferencias significativas ( $\alpha \leq$ 0,06) (Tabla 3). Estos resultados coinciden con estudios realizados en bosques del centro de México y noroeste de Estados Unidos, donde se encontró que la

Tabla 2. Coeficientes Canónicos Estandarizados (CCE) y variables que influyen sobre la calidad de estación en el sur de Chihuahua, México.

\begin{tabular}{ccc}
\hline Variable & CAN1 & CAN2 \\
\hline Asnm & 0,18443 & $-0,34208$ \\
Pendiente & $-0,26774$ & 0,23286 \\
Profundidad de suelo & 0,42830 & 0,15509 \\
Pedregosidad & $-0,27038$ & 0,08543 \\
Suelo desnudo & 0,09848 & $-0,10904$ \\
Grosor de mantillo & 0,26814 & 0,01415 \\
Hojarasca & 0,10773 & $-0,02531$ \\
Disección horizontal & 0,11665 & 0,26618 \\
Disección vertical & $-0,59968$ & 0,27544 \\
Exposición & $-0,42088$ & $-0,10468$ \\
Temperatura mínima & 0,22402 & 0,59289 \\
Precipitación & 0,49133 & 0,23916 \\
\hline
\end{tabular}

Variable canónica 1 (CAN1) dada por pendiente, profundidad de suelo, disección vertical, temperatura máxima y precipitación y la variable canónica 2 (CAN 2) por temperatura mínima, disección horizontal. 


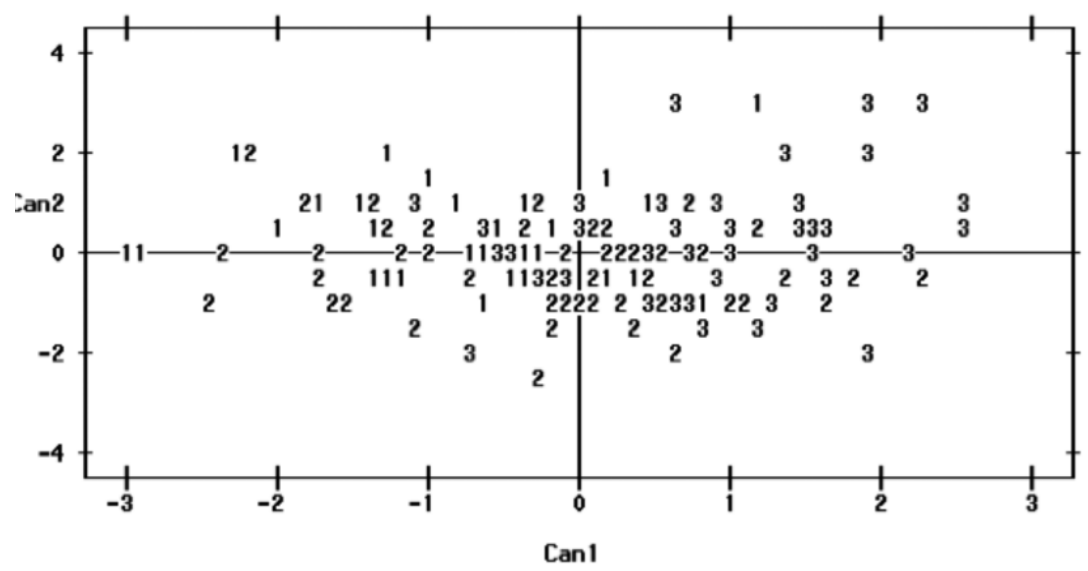

Figura 4. Separación de las variables canónicas.

Separación de grupos a partir de valores canónicos de calidad de estación. Los niveles corresponden a $1=$ Calidad de estación mala, 2 = Calidad de estación media y 3 = Calidad de estación alta.

productividad se ve afectada por la pendiente, exposición y profundidad de suelo (Griffiths et al., 2009; Gómez Tagle, 1985; Chávez y Gómez Tagle, 1985).

Las variables pedregosidad, porcentaje de suelo desnudo, grosor de mantillo, grosor de hojarasca y precipitación no mostraron diferencias significativas ( $\alpha \leq$ $0,05)$ entre las calidades de estación (Tabla 3). Esto puede deberse a la complejidad del territorio estudiado o a las actividades de intenso manejo que se desarrollan en el área de estudio, las cuales podrían modificar las características del suelo, del material orgánico sobre el suelo y del material en descomposición (Nave et al., 2010; Poeplau et al., 2011; Vincent et al., 2010). Aunque diversos estudios han mostrado que los componentes del suelo ejercen una marcada influencia en la productividad del sitio y el desarrollo del arbolado; por ejemplo, Mader (1976) menciona que las características del suelo explican alrededor de $60 \%$ de la productividad, mientras que
Sánchez y Chacón (1986) encontraron diferencias notables en rocosidad y profundidad de suelo en relación con la productividad de Pinus arizonica en el norte de Chihuahua, México, por su parte Narváez (1990) encontró que el pH, el grosor de mantillo y las características del horizonte A influyen sobre el potencial productivo del bosque. Sin embargo, a diferencia de las variables de clima y relieve, las variables de suelo pueden ser afectadas fuertemente por disturbios naturales o por factores antropogénicos como las cosechas y el manejo (Heiberg y Whithe, 1956), lo cual dificulta la identificación de influencia de macrovariables, sobre todo en estudios de tipo regional. Ante esto, se han aplicado estrategias como el uso de variables que caracterizan al relieve para definir características del suelo. Ésta ha sido una práctica útil para caracterizar suelos en extensas regiones (Thiers y Gerding, 2007).

La tabla 4 muestra los valores medios para cada variable en cada calidad de 
Tabla 3. Análisis de varianza comparación de variables ecológicas entre sitios (productividad alta, media y baja)

\begin{tabular}{|c|c|c|c|c|}
\hline Variable & $\begin{array}{l}\text { Suma de } \\
\text { cuadrados }\end{array}$ & Cuadrado medio & $F_{C}$ & Significancia \\
\hline Altura sobre el nivel del mar (m) & 461386,09467 & 230693,04733 & 2,23822 & 0,109659 \\
\hline Pendiente (grados) ${ }^{\star *}$ & 0,21467 & 0,10734 & 3,99936 & 0,020047 \\
\hline Profundidad de suelo $(\mathrm{cm})^{*}$ & 527,35401 & 263,67700 & 0,69847 & 0,012343 \\
\hline Pedregosidad (\%) & 0,15431 & 0,07716 & 2,33223 & 0,100090 \\
\hline Suelo desnudo (\%) & 0,00240 & 0,00120 & 0,05643 & 0,945152 \\
\hline Grosor de mantillo $(\mathrm{cm})$ & 4,31110 & 2,15555 & 2,03871 & 0,133350 \\
\hline Grosor de hojarasca $(\mathrm{cm})$ & 0,07070 & 0,03535 & 0,64367 & 0,526795 \\
\hline Disección horizontal $\left(\mathrm{m} / \mathrm{km}^{2}\right)$ & 665331,51108 & 332665,75554 & 0,59109 & 0,554842 \\
\hline Disección vertical $\left(\mathrm{m} / \mathrm{km}^{2}\right)^{\star *}$ & 170835,01070 & 85417,50535 & 11,22476 & 0,000026 \\
\hline Exposición* & 5,71505 & 2,85753 & 2,82671 & 0,061883 \\
\hline Temperatura mínima $\left({ }^{\circ} \mathrm{C}\right)^{* *}$ & 37,37838 & 18,68919 & 3,26125 & 0,040670 \\
\hline Temperatura máxima $\left({ }^{\circ} \mathrm{C}\right)^{* *}$ & 86,15165 & 43,07583 & 5,20779 & 0,006349 \\
\hline Precipitación $(\mathrm{mm})$ & 1219,02285 & 609,51142 & 0,04307 & 0,0957852 \\
\hline
\end{tabular}

estación. Estos valores pueden ser utilizados para crear modelos espaciales (mapas) para la elaboración de un modelo general de productividad en la región de estudio. La temperatura mínima y la temperatura máxima mostraron sensibles diferencias. Por su parte, no se identificaron diferencias significativas en el análisis para la variable precipitación.

Al respecto, Anaya et al. (1980) señalan que las principales variables que influyen sobre el crecimiento en altura y diámetro de las coníferas son las climáti- cas, como las temperaturas extremas y la precipitación. No obstante, en territorios con homogeneidad en las variables climáticas, como en el caso de la región de estudio, es complicado encontrar diferencias significativas.

Los valores de las variables climáticas (Tabla 4) también pueden ser usados para reclasificar modelos espaciales para definir regiones con diferente productividad. Estos datos podrían sumarse a las variables de relieve y suelo para desarrollar un modelo espacial de productividad. 
Tabla 4. Medias y desviaciones estándar de las variables físicas por sitios (productividad alta, media y baja)

\begin{tabular}{|c|c|c|c|c|c|c|}
\hline \multirow{2}{*}{ Variable } & \multicolumn{2}{|c|}{ P. Baja } & \multicolumn{2}{|c|}{ P. Media } & \multicolumn{2}{|c|}{ P. Alta } \\
\hline & Media & Dev. Std. & Media & Dev. Std. & Media & Dev. Std. \\
\hline Pendiente (grados) & 37 & 15 & 30 & 15 & 12 & 8 \\
\hline Profundidad de suelo $(\mathrm{cm})$ & 25,82 & 17,49 & 35,48 & 18,73 & 43,73 & 11,05 \\
\hline Pedregosidad (\%) & 0,29 & 0,2 & 0,22 & 0,17 & 0,21 & 0,18 \\
\hline Suelo desnudo (\%) & 0,12 & 0,1 & 0,13 & 0,14 & 0,13 & 0,17 \\
\hline Grosor de mantillo $(\mathrm{cm})$ & 1,51 & 0,84 & 1,64 & 0,84 & 1,9 & 1,27 \\
\hline Grosor de hojarasca $(\mathrm{cm})$ & 0,58 & 0,22 & 0,63 & 0,22 & 0,64 & 0,25 \\
\hline Disección horizontal $\left(\mathrm{m} / \mathrm{km}^{2}\right)$ & 1833,65 & 781,46 & 1704,69 & 730,78 & 1828,79 & 752,79 \\
\hline Disección vertical $\left(\mathrm{m} / \mathrm{km}^{2}\right)$ & 311,23 & 90,86 & 238,51 & 84,51 & 234,25 & 87,89 \\
\hline Exposición (rumbo) & S, SE & & $\begin{array}{c}\text { NW, } \\
\text { s y SW }\end{array}$ & & $\mathrm{N}, \mathrm{NW}$ y $\mathrm{Z}$ & \\
\hline Temperatura mínima $\left({ }^{\circ} \mathrm{C}\right)$ & 5 & 2 & 4 & 1 & 8 & 3 \\
\hline Temperatura máxima $\left({ }^{\circ} \mathrm{C}\right)$ & 23 & 3 & 23 & 2 & 18 & 3 \\
\hline Precipitación (mm) & 794 & 43 & 897 & 92 & 901 & 116 \\
\hline
\end{tabular}

\section{CONCLUSIONES}

El uso de modelos de índice de sitio permitió definir niveles de productividad en la zona de distribución de Pinus arizonica y $P$. engelmannii, así como identificar las variables con mayor relación sobre la productividad de los sitios en la región sur del estado de Chihuahua.

Los análisis de discriminante canónico y de varianza identificaron diferencias significativas en las variables físicas (profundidad de suelo, pendiente, disección vertical, temperatura mínima y tempera- tura máxima). Los promedios e intervalos de estas variables pueden ser de gran utilidad para el desarrollo de cartografía de potencial productivo en la región de estudio.

Las variables pedregosidad, porcentaje de suelo desnudo, grosor de mantillo y grosor de hojarasca, no mostraron diferencias significativas entre los estratos con diferente productividad. Esto puede deberse a que son variables fáciles de modificar por acciones de manejo de los recursos. 


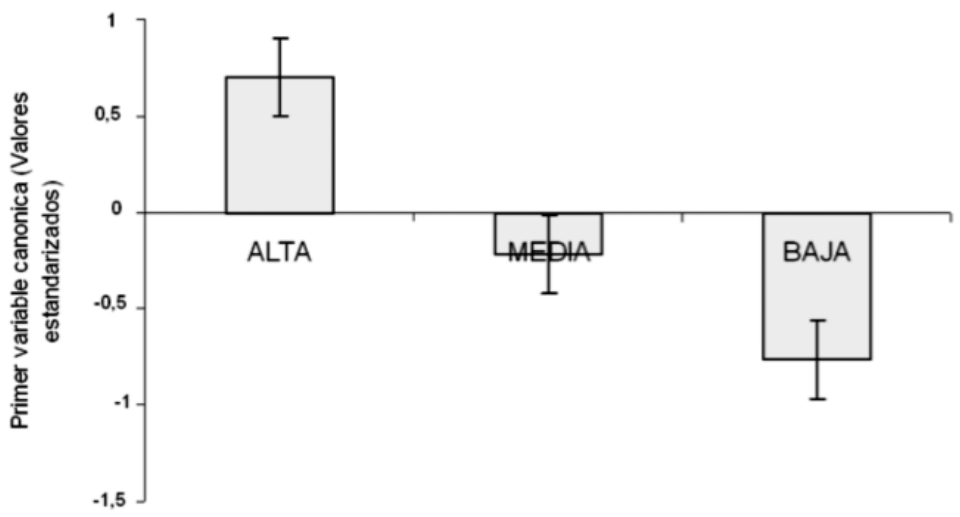

Calidades de estación

Figura 5. Diferencias multivariadas por calidad de estación

\section{RECONOCIMIENTOS}

Se agradece al fondo sectorial ConaforConacyt, quien financia el proyecto "Estudio para determinar el potencial productivo de bosques templados" (C03-10027) y a los silvicultores del sur de Chihuahua por el apoyo brindado para desarrollar el trabajo de campo.

\section{REFERENCIAS}

Anaya, L.A., S.R. Hernández y H.S. Madrigal. 1980. La vegetación y los suelos de un transecto altitudinal del declive occidental del Iztaccíhuatl. Bol. Tec. 65. INIF, SFF, México. 79 p.

Castaños, M.L. 1962. Evaluación de la calidad de estación de Pinus patula en el norte de Oaxaca. Bol. Tec. INIF 2. $32 \mathrm{p}$.

CETENAL (Centro de Estadísticas del Territorio Nacional). 1972. Carta de climas Esc. 1:250000 CETENAL, México. $1 \mathrm{p}$.
Chávez, H.M.Y.M. y R.AF. Gómez-Tagle. 1985. Principales interacciones entre los suelos forestales y las coníferas del Cerro de la Cruz. Mich. Bol. Téc. núm. 10, INIF, México $32 \mathrm{p}$.

Clutter, J.L., J.C. Fortson, L.V. Pienaar y R.L. Bailey. 1983. Timber management: A quantitative approach. Wiley. Nueva York. 333 p.

Daniel, T.W., J.A. Helms y F.S. Baker. 1982. Principios de silvicultura. McGraw Hill. México. 492 p.

Deo-Singh, K. 2013. Estimating potential productivity of forests. Environmental Science and Ingeniering. p:147-158.

Eguiluz, P.T. 1977. Los pinos del mundo, Escuela Nacional de Agricultura. Chapingo, México. Publicación especial núm.1. $75 \mathrm{p}$.

Eguiluz, P.T. 1982. Clima y distribución del género Pinus en México. Revista Ciencia Forestal en México 38(7): 30-44. 
FPC (Forest Productivity Council) B.C. 1998. Minimum Standards and Stem Analysis Procedures for Site Index Research. Victoria, BC. 9 p.

García, E. 1981. Modificaciones al sistema de clasificación climática de Köeppen, (para adaptarlo a las condiciones de la República Mexicana), UNAM, 3a. ed, México, D.F.

Gómez-Tagle, R.AF. 1985. Levantamiento de suelos del Campo Experimental Forestal Barranca de Cupatitzio y sus relaciones con la vegetación de coníferas, Tesis de maestría, Fac. Ciencias, UNAM, México. 135p.

Griffiths, R.P., M.D. Madritch y A.K. Swanson. 2009. The effects of topography on forest soil characteristics in the Oregon Cascade Mountains (USA): Implications for the effects of climate change on soil properties. Forest Ecology and Management 257(1): 1-7.

Hair, J.F.Jr., R.E. Anderson, R.L. Tatham y W.C. Black. 1998. Multivariate data analysis. 5a. ed. Prentice-Hall. EUA.

Heiberg, S.O. y D.P. White. 1956. A site evaluation concept. Journal of forestry 54:7-10.

IMTA (Instituto Mexicano de Tecnología del Agua). 2006. Sistema de bases de datos meteorológicas. ERIC Ver. 3. México.

Karhu, K., H. Fritze M. Tuomi, P. Vanhala, P. Spetz, V. Kitunen y J. Liski. 2010. Temperature sensitivity of organic matter decomposition in two boreal forest soil profiles. Soil Biology and Biochemestry 42 (1):72-82.

Latta, G., H. Temesgen, D. Adams y T. Barrett. 2010. Analysis of potential impacts of climate change on forests of the United States Pacific Northwest. Forest Ecology and Management 259(4):720-729.

Latta, G., H. Temesgen y T.M. Barret. 2009. Mapping and imputing potential productivity of Pacific Northwest forests using climate variables. Canadian Journal of Forest Research 39(6):1197-1207.

Mader, D.F. 1976. Soil-site productivity for natural stands of white pine in Massachusetts. Soil Science Society American Journal 40:112-115.

Madrigal, H.S. 2002. Evaluación del potencial productivo de sitio forestal para Pinus oocarpa en el ejido Patuan, municipio de Ziracuaretiro, Michoacán. In: IV Congreso Forestal Mexicano. Morelia, Michoacán. Asociación de Profesionales Forestales A.C. $1 \mathrm{p}$.

Martínez, S.M., R.A. Olivas, R.V. Cepeda y L.B. Morales. 2006. Clasificación de potenciales naturales en los bosques templados del sur de Chihuahua. Folleto Técnico núm. 35. Instituto Nacional de Investigaciones Forestales, Agrícolas y Pecuarias, México. 33 p.

Martínez, S.M. 2008. Potencial productivo y zonificación forestal para el reordenamiento silvícola en bosques templados. Folleto Técnico núm. 37. Inifap, C.E. La Campana. Chihuahua, Chih., Méx. 52 p.

Mas P.J. 1970. Instructivo para realizar análisis troncales. Bol. Div. Inst. Nal. de Invest. Ftales núm. 23. México. $23 \mathrm{p}$.

Medlyn, B.E., R.A. Duursma y M.J.B. Zeppel. 2011. Forest productivity under 
climate change: a checklist for evaluating model studies. Wiley Interdisciplinary Reviews: Climate Change 2(3):332-355.

Musalem, S.M., C. Rodríguez, F. Carrillo y G. Vera. 1986. Proyecciones de la investigación silvícola en los suelos forestales de México. INIFAP. Inédito $25 \mathrm{p}$.

Narváez, F.R. 1990. El suelo en relación con los tipos de vegetación y su productividad en el área experimental forestal Madera, Chihuahua. Tesis de maestría. Universidad Nacional Autónoma de México. Facultad de Ciencias. México, D.F. 109 p.

Nave, L.E., E.D. Vance, C.W. Swanston y P.S. Curtis. 2010. Harvest impacts on soil carbon storage in temperate forests. Forest Ecology and Management 259(5):857-866.

Palahí, M., M. Tome, T. Pukkala, A. Trasobares y G. Montero. 2004. Site index model for Pinus sylvestris in north east Spain. Forest Ecology and Management (187):35-47.

Peters, E.B., K.R. Wythers, J.B. Brandford y P.B. Reich. 2013. Influence of disturbance on temperate forest productivity. Ecosystems 16(1):95-110.

Poeplau, C., A. Don, L. Vesteral, J. Leifeld, B.V. Wesemael, J. Schumacher y A. Gensior. 2011. Temporal dynamics of soil organic carbon after land-use change in the temperate zone-carbon response functions as a model approach. Global Change Biology 17(7):2415-2427.

Pritchett, L.W. 1986. Suelos forestales (propiedades, conservación y mejoramiento). Limusa. México. $634 \mathrm{p}$.
Raz-Yaseef, N., E. Rotenberg y D. Yakir. 2010. Effects of spatial variations in soil evaporation caused by tree shading on water flux partitioning in a semi-arid pine forest. Agricultural and Forest Meteorology 150(3):454-462.

Rodríguez, F.C. 1982. Determinación de la calidad de estación de Pinus montezumae Lamb, a través de análisis troncales en el CEF San Juan Tetla, Puebla. Tesis de maestría. Colegio de Postgraduados, Chapingo, México. $134 \mathrm{p}$.

Rzedowski, R.J. 1978. Vegetación de México. Limusa. México, D.F. 432 p.

Sánchez, C.J. y S.J. Chacón. 1986. Relación suelo-vegetación del área experimental forestal madera, INIFAP. Ciencia Forestal 11(59):41-64.

Spurr, S.H. 1952. Forest Inventory. Ronald Press. Nueva York, EUA. 476 p.

Spurr, S.H. y B.V. Barnes. 1982. Ecología Forestal, México. AGT, Editor. 690 p.

Stoler, A.B. y R.A. Relyea. 2011. Living in the litter: the influence of tree leaf litter on wetland communities. Oikos 120(6):862-872.

Tellez, V.O., H.MYM. Chávez, R.A.F. Gómez-Tagle y G.M. Gutiérrez. 2004. Modelado bioclimático como herramienta para el manejo forestal: Estudio de cuatro especies de Pinus. Revista Ciencia Forestal en México 95(29):61-82.

Thiers, O. y V. Gerding. 2007. Variabilidad topográfica y edáfica en bosques de Nothofagus betuloides (Mirb) Blume, en el suroeste de Tierra del Fuego, Chile. Revista chilena de historia natural 2(80):201-211. 
Vincent, A.G., B.L. Turner y E.V.J. Tanner. 2010. Soil organic phosphorus dynamics following perturbation of litter cycling in a tropical moist forest. European Journal of Soil Science 61(1):48-57. dad de Pinus arizonica y Pinus engelmannii en el sur de Chihuahua, Méx. Madera y Bosques 19(3):35-49. 